\title{
El Significado de la Negación Paraconsistente
}

\author{
GLADYS PALAU \\ Universidad de Buenos Aires/Universidad Nacional de La Plata \\ CECILIA DuRAN \\ Universidad Nacional de La Plata
}

\begin{abstract}
This work agrees and supports the I. Hacking's thesis regarding the meaning of the logical constants accordingly with Gentzen's Introduction and Elimination Rules of Sequent Calculus, corresponding with the abstract conception of the notion of logical consequence. We would like to ask for the minimum rules that must satisfy a connective in order to be considered as a genuine negation. Mainly, we will refer to both da Costa's C-Systems and Priest's LP system. Finally, we will analyze the presentations of these systems within the Sequent Logic to show that paraconsistent negation lacks of pure rules of negation-elimination and negation-introduction rules or that they involve other connectives, thus making difficult to assign an univocal meaning to paraconsistent negation.
\end{abstract}

Keywords: Paraconsistent logic, negation, sequent calculus.

\section{Introducción}

En un artículo fundacional What is Logic? (1979), Ian Hacking, arroja luces decisivas sobre el problema clásico en filosofía de la lógica tal como lo es el significado de las constantes lógicas. Hacking comienza definiendo la lógica como transiciones de oraciones a otras oraciones. Estas transiciones deben expresarse en el metalenguaje y deben considerarse solamente descripciones o codificaciones de cómo nosotros creemos que deben hacerse las mismas cuando deseamos decir que ellas son de carácter lógico. Además, pueden entenderse como casos especiales de transformaciones y pueden ser pensadas tanto semántica como sintácticamente y, de acuerdo con Wittgenstein, nunca pueden calificarse de justificaciones. Para ello adopta el cálculo de secuentes de Gentzen, en el cual las reglas estructurales caracterizan el concepto abstracto de consecuencia lógica mientras que las reglas operatorias caracterizan el uso o significado de las constantes lógicas en concordancia con las reglas estructurales. A su vez, las reglas operatorias que caracterizan las constantes lógicas sólo tienen sentido leídas en el marco de deducibilidad generado por las reglas estructurales y sólo dentro de esta estructura de conjunto puede decirse que constituyen el significado de las constantes lógicas. Esta tesis ha sido rigurosamente fundamentada por R. Wójcicki (1988) quien sostiene que toda operación de consecuencia está

Principia 13(3): 357-70 (2009).

Published by NEL — Epistemology and Logic Research Group, Federal University of Santa Catarina (UFSC), Brazil. 
unívocamente determinada por el conjunto de reglas de inferencia consistentes con ella y muestra que hay lógicas que coinciden en el conjunto de sus teoremas pero no coinciden en el conjunto de sus reglas de inferencia, tal como es el caso de la lógica intuicionista y de la lógica de la relevancia respecto de la lógica clásica. ${ }^{1} \mathrm{Es}$ precisamente dentro de este enfoque que analizaremos el significado de la negación en la lógica paraconsistente ya que actualmente se acuerda que ella es una lógica subclásica. Hoy en día se acepta en considerar que una lógica es paraconsistente si y sólo si no satisface la regla conocida con el nombre de Ex Contradictione Sequitur Quodlibet. $^{2}$

$$
\Gamma, A, \neg A \Rightarrow B, \Delta \quad \text { (ECQ). }
$$

Es decir, los sistemas de lógica paraconsistente son aquellos que admiten contradicciones pero no por ello originan sistemas triviales, lo cual implica distinguir nítidamente inconsistencia de trivialidad. En palabras de W. A. Carnielli y J. Marcos (2007: 11), una lógica es paraconsistente si es inconsistente pero no trivial. Coincidimos con estos autores en que uno de los argumentos más fuertes a favor de la importancia de estos sistemas sea la existencia efectiva de teorías inconsistentes pero no triviales, como por ejemplo, la teoría intuitiva de conjuntos.

En el enfoque de Hacking el significado de las conectivas lógicas, incluso el de la negación, depende de las reglas admitidas o rechazadas que rigen el uso de cada conectiva. Precisamente el Cálculo de Secuentes de Gentzen nombra las reglas que rigen la introducción y la eliminación de las conectivas como Introducción en el postsecuente y en el prosecuente respectivamente. Por ello, dada una regla que no debe quedar validada por la teoría, hay que explorar qué es lo que se debe alterar en el cálculo de secuentes a fin de filtrarla. En el caso que nos ocupa, dado que el rechazo de ECQ es común a todo sistema de lógica paraconsistente, todo sistema paraconsistente deberá rechazar también las reglas que permiten derivar ECQ. En particular, desde el marco de la lógica de secuentes, se deberían rechazar o modificar las reglas estructurales o las operatorias que posibilitan la derivación de tal regla.

Si se explora en esta lógica qué reglas permiten la demostración de ECQ, se encuentra que la demostración en lógica de secuentes de ECQ involucra Introducción de la Negación en el prosecuente $(\neg \Rightarrow)$, Introducción de la Conjunción en el prosecuente $(\wedge \Rightarrow)$ y Atenuación en el postsecuente $\left(\Rightarrow\right.$ At). ${ }^{3}$ Por ello, se abren dos caminos, o bien se pone en duda ( $\Rightarrow$ At) o bien falla $(\neg \Rightarrow)$. Exploraremos ambas alternativas.

La primera opción no resulta conveniente ya que si se descarta $(\Rightarrow \mathrm{At})$ se restringe la potencia lógica de los sistemas paraconsistentes, puesto que esta regla es necesaria para la demostración en lógica de secuentes del axioma del sistema $\mathrm{C}_{\omega}$ de N. C. A. da Costa, D. Krause y O. Bueno (2007: 798), $(A \rightarrow C) \rightarrow((B \rightarrow C) \rightarrow$ $((A \vee B) \rightarrow C))$ y para la derivación de la Ley de Dummett, $(A \vee(A \rightarrow B)) .{ }^{4}$ Además, 
quitar $(\Rightarrow \mathrm{At}$ ) no es condición suficiente para evitar explosión, ya que ello no impide la derivación de otra forma de explosión, como es el caso de la regla $\Gamma, A, \neg A \Rightarrow \neg B, \Delta$ cuya derivación no emplea ( $\Rightarrow \mathrm{At}$ ).

La segunda opción nos conduce a explorar qué sucede en una lógica paraconsistente con la regla operatoria de Introducción de la negación en el prosecuente $(\neg \Rightarrow)$. Si esta regla fallara en todas sus aplicaciones, la desviación de la lógica paraconsistente respecto de la lógica clásica no estaría dada por las reglas estructurales sino por alguna de las reglas operatorias que involucran la negación, lo cual condicionaría el significado de esta conectiva en la mencionada lógica. Elucidar estas cuestiones y sus consecuencias para la filosofía de la lógica es uno de nuestros objetivos principales.

Para nuestro trabajo hemos elegido como referencia los sistemas que consideramos más representativos de este tipo de lógica en la literatura actual, a saber, los C-sistemas de N. C. A. da Costa et al. (2007), y el sistema LP de Graham Priest (2001), ya que ellos emplean dos estrategias totalmente diferentes, la vía de una lógica no veritativo funcional por parte de la escuela de da Costa y la vía de una lógica multivaluada por parte de Priest.

\section{La Negación en los C-sistemas de da Costa et al.}

Es sabido que en todo sistema lógico paraconsistente, a los efectos de obtener sistemas no triviales pero que admitan contradicciones se debe satisfacer las reglas de las conectivas positivas pero no debe satisfacerse la ya citada regla ECQ. Respecto del Principio de No Contradicción la situación es distinta ya que si se desea que el sistema sea una lógica dialéctica, como el sistema DL de da Costa-Wolf (1980) este principio no puede ser válido, de donde se sigue que su rechazo no constituye una condición necesaria para la paraconsistencia. Los sistemas paraconsistentes de da Costa conforman una colección jerárquica $\mathrm{C}_{n}, 0 \leq n \leq \omega$ construida a partir de los axiomas positivos de la axiomática de Hilbert. $\mathrm{C}_{\omega}$ contiene la negación paraconsistente más débil y por ello está caracterizado solamente por 11 axiomas, entre los que se incluye el Modus ponens (MP) en tanto regla y dos axiomas específicos para la negación: la doble negación no intuicionista $\neg \neg A \rightarrow A$ (DN) y el principio clásico Tertium non datur $A \vee \neg A$ (TND). Como en todo C-sistema, además de ECQ, en $\mathrm{C}_{\omega}$ tampoco puede ser válida la regla clásica Reductio ad absurdum (RAb), $A \rightarrow B$, $A \rightarrow \neg B \Rightarrow \neg A$ debida a Johanssen y Kolmorogov y tan exitosamente usada en la matemática, porque si valiera, sería posible demostrar $\neg(A \wedge \neg A)$ como teorema por Sustitución Uniforme a partir de los dos primeros axiomas positivos de $C_{n}$ con dos aplicaciones de MP (Maranhão 2001: 13). ${ }^{5}$

Tampoco puede valer el principio de doble negación intuicionista $A \Rightarrow \neg \neg A$, porque si valiera conjuntamente con el axioma $\neg \neg A \Rightarrow A$, la negación paraconsistente 
colapsaría nuevamente en la negación clásica. La falla de la regla (RAb) hace caer todas las formas de la Transposición e incluso al Silogismo Disyuntivo (SD) ${ }^{6}$ y al Modus tollens. Tampoco resultan teoremas $\neg A \rightarrow(A \rightarrow B), \neg A \rightarrow(A \rightarrow \neg B), A \rightarrow(\neg A \rightarrow B)$, $A \rightarrow(\neg A \rightarrow \neg B),(A \wedge \neg A) \rightarrow B)$ y $(A \wedge \neg A) \rightarrow \neg B)$ cuyas demostraciones requieren de ECQ. Dado que en lógica de secuentes todas estas reglas utilizan en su demostración la regla Introducción de la negación en el prosecuente $(\neg \Rightarrow)$, se podría entonces suponer que en todo sistema paraconsistente se debería descartar esta regla. Sin embargo esta alternativa plantea los siguientes dos problemas (i) si se aplica Sustitución Uniforme en el primer axioma positivo de $\mathrm{C}_{\omega}$, i.e., $\Rightarrow A \rightarrow(B \rightarrow A)$, entonces resultan teoremas los casos de Causa mirabilis $\neg A \rightarrow(A \rightarrow \neg A)$ y $A \rightarrow(\neg A \rightarrow A)$, cuyas demostraciones en lógica de secuentes involucran la regla $(\neg \Rightarrow)$ y (ii) la demostración en lógica de secuentes del axioma de $\mathrm{C}_{\omega}, \neg \neg A \rightarrow A$ requiere tanto de Introducción de la negación en el postsecuente $(\Rightarrow \neg$ ) como de la regla de Introducción en el prosecuente $(\neg \Rightarrow)$. Sin embargo se debe tener en cuenta que si se descarta la regla $(\neg \Rightarrow)$ entonces todas las formas de explosión quedan obstruidas, lo cual muestra la utilidad de su rechazo. Por otra parte, es posible resolver los inconvenientes planteados en (i) y (ii) prescindiendo de $(\neg \Rightarrow)$. En efecto, los casos de Causa mirabilis planteados en (i) quedan validados en los C-sistemas si se admite que las fórmulas fundamentales o axiomas de los que parte toda derivación, puedan ser literales (i.e., fórmulas atómicas o fórmulas atómicas negadas) del tipo $\neg A \Rightarrow \neg A^{7}$ y además se acepte atenuar con literales. En relación con el inconveniente (ii) i.e., el referido a la Eliminación de Doble Negación, Andrés Raggio (1968) realiza un análisis de la negación en los sistemas $\mathrm{C}_{\omega}$ desde la lógica de Secuentes y propone caracterizar la negación paraconsistente de $\mathrm{C}_{\omega}$ por la siguiente regla de Introducción de la doble negación en el prosecuente $(\neg \neg \Rightarrow)$ la cual se conserva en todos los C-sistemas, a saber:

$$
\frac{A, \Gamma \Rightarrow \Theta}{\neg \neg A, \Gamma \Rightarrow \Theta}
$$

En el cálculo de Raggio para $\mathrm{C}_{\omega}$, además de la regla de la doble negación se efectúan dos modificaciones más: (i) se elimina la regla $(\neg \Rightarrow)$ de la lógica de secuentes de Gentzen, dejando solamente la mencionada $(\neg \neg \Rightarrow)$ y (ii) se modifica la regla $(\Rightarrow \rightarrow)$ de Gentzen mediante una restricción según la cual en el postsecuente de la premisa sólo puede aparecer la fórmula que es el consecuente del condicional que a su vez es conclusión de la regla. O sea, la regla $(\Rightarrow \rightarrow)_{R}$ :

$$
\frac{A, \Gamma \Rightarrow B}{\Gamma \Rightarrow A \rightarrow B}
$$

Esta modificación filtra perfectamente tanto la ley de Dummet como la ley de Peirce que no son teoremas de $\mathrm{C}_{\omega}$ dado que éste fue construido sobre el fragmento positivo del sistema de Hilbert, según lo afirman sus propios autores. Además, 
$(\Rightarrow \rightarrow)_{R}$ no cumple con toda la restricción intuicionista (i.e. que el postsecuente no pueda tener más de una fórmula) ya que si lo hiciera quedaría comprometida la validez de los axiomas para la negación de $\mathrm{C}_{\omega}$.

Corresponde preguntarnos ahora qué resultados se siguen de nuestro análisis a los efectos de caracterizar la negación en $\mathrm{C}_{\omega}$. Ya hemos afirmado que en $\mathrm{C}_{\omega}$ no se valida ECQ pero sí DN y TND. Sin embargo, de estos axiomas no se sigue ninguna otra propiedad interesante respecto de la negación en $\mathrm{C}_{\omega}$. Por ello, ahí que lo único que parece caracterizar positivamente un sistema tan débil respecto de la negación como $C_{\omega}$ es que $C_{\omega}$ no es finitamente trivializable (da Costa et al. 2007: 809). Sin embargo, a partir de la enumeración ya dada de las reglas que no se satisfacen en $\mathrm{C}_{\omega}$, es posible aventurar una caracterización negativa de la negación. Otra posibilidad consiste en encontrar reglas mínimas o puras con las que se pueda determinar el significado de la negación en $\mathrm{C}_{\omega}$. La primera alternativa nos enfrenta al problema lógico filosófico de si es posible inferir lo que cierta cosa es partiendo solamente de lo que ella no es, dado que en una metateoría clásica sigue valiendo el dictum aristotélico de que no hay inferencias válidas con todas sus premisas negativas.

Una caracterización precisa de la negación en $\mathrm{C}_{\omega}$ debería también tener en cuenta ciertas peculiaridades. $\mathrm{C}_{\omega}$ carece de una negación fuerte, es decir no es definible como $\neg \neg^{(n)} A=\mathrm{df} \neg A \wedge A^{(n)}$. Además, si bien $\mathrm{C}_{\omega}$ no es finitamente trivializable, es posible definir una negación a partir del fragmento implicativo de $\mathrm{C}_{\omega}$ siempre que el conjunto de fórmulas de $\mathrm{C}_{\omega}$ se construya con elementos de un conjunto finito de variables proposicionales ${ }^{8}$ (Maranhão 2001: 29). Resulta sencillo advertir que al carecer de la regla $(\neg \Rightarrow)$ no se valida Introducción de de la Doble Negación en el postsecuente $(\Rightarrow \neg \neg)$ ni tampoco es posible validar teoremas negados o probar equivalencia de fórmulas negadas.

La escuela de da Costa y él mismo entendieron la debilidad del sistema $\omega$ desde un principio y por ello, para construir la jerarquía de los C-sistemas, en particular $\mathrm{C}_{1}$, adicionan un nuevo operador denominado estabilizador para "estabilizar" fórmulas y simbolizado por ${ }^{\circ}$ de forma tal que $A^{\circ}={ }_{\mathrm{df}} \neg(A \wedge \neg A)$ y dos nuevos axiomas, uno que garantiza que las fórmulas compuestas por fórmulas estabilizadas también "se portan bien", llamado Propagación del Buen Comportamiento $A^{\circ} \wedge B^{\circ} \rightarrow\left(A^{\circ} \wedge B^{\circ}\right) \wedge$ $\left(A^{\circ} \vee B^{\circ}\right) \wedge\left(A^{\circ} \rightarrow B^{\circ}\right)$ y otro que dota a la negación de nuevas propiedades $B^{\circ} \rightarrow(A \rightarrow$ $B) \rightarrow((A \rightarrow \neg B) \rightarrow \neg A)$ ). Este último axioma mediante el converso del Metateorema de a Deducción, se traduce en la regla conocida como Absurdo Paraconsistente (PAb).

Dado que la invalidez de (RAb) en $\mathrm{C}_{\omega}$ precisamente impedía todas las formas de explosión, (PAb) debería conservar esa propiedad. Efectivamente, (PAb) expresa la explosión frente a fórmulas que respetan el Principio de No Contradicción pero no para fórmulas cualesquiera. Así, la negación de una fórmula estabilizada es una negación fuerte en el sentido de que es explosiva. No es necesario discriminar la graduación de propiedades de la negación en los diversos sistemas $\mathrm{C}_{n}$, pero sí se 
requiere aclarar que $A^{n}$ abrevia fórmulas con un grado de complejidad creciente, siendo que ello hace variar el tipo de fórmula que trivializa esos sistemas. O sea, a medida que incrementa $n$ en $C_{n}$ tal que $0 \leq n \leq \omega$ la lógica se debilita, $C_{0}$ es la lógica clásica y $\mathrm{C}_{1}$ el sistema más potente dentro de la jerarquía de cálculos paraconsistentes. Analizaremos pues la negación en $\mathrm{C}_{1}$ a fin de completar lo expresado respecto de ella. $\mathrm{Al}$ adicionar (PAb) a $\mathrm{C}_{1}$, se obtienen las reglas clásicas para la negación pero a condición de que queden estabilizadas las fórmulas que producen explosión. Sin embargo en $\mathrm{C}_{1}$ sigue sin ser válido el Silogismo disyuntivo $\mathrm{y}$, a menos que $\mathrm{A}$ esté estabilizada tampoco es teoremas la fórmula $(A \vee B \rightarrow(\neg A \rightarrow B)$. Más aún, también se satisface en $\mathrm{C}_{1}$ la regla según la cual, $A^{\circ}, A, \neg A \Rightarrow B$. Debemos preguntarnos entonces qué reglas en secuentes quedan involucradas en la demostración de (PAb). La solución nos lleva a reintroducir en el cálculo de secuentes la negación en el prosecuente que fuera cancelada para $\mathrm{C}_{\omega}$. Pero, si se introduce la regla de Gentzen, la negación paraconsistente colapsaría con la clásica. Para evitar esto es suficiente emplear la regla $\left({ }^{\circ} \neg \Rightarrow\right)$ (Benassi et al. 2007).

$$
\frac{A^{\circ}, \Gamma \Rightarrow \Theta, A}{A^{\circ}, \neg A, \Gamma \Rightarrow \Theta} \quad\left({ }^{\circ} \neg \Rightarrow\right)
$$

Aplicando $\left({ }^{\circ} \neg \Rightarrow\right)$ es derivable (PAb) en el Cálculo de Secuentes. ${ }^{9}$ Nótese que esta modificación no reintroduce Pseudo Scotus ni Explosión puesto que A está estabilizada. Además, es posible redefinir la regla para cada uno de los $C_{n}$ en función de la complejidad de las fórmulas para las que vale ${ }^{\circ}$. Para completar el cálculo de secuentes se deberían construir reglas para la introducción del estabilizador, bajo la guía de la definición de $A^{\circ}$ como $\neg(A \wedge \neg A)$ de modo que se cumpla $\Rightarrow A^{\circ} \rightarrow \neg(A \wedge \neg A)$ $\mathrm{y} \Rightarrow \neg(A \wedge \neg A) \rightarrow A^{\circ}$.

Agregando la definición de la negación fuerte, i.e., $\neg^{*} A=_{\mathrm{df}} \neg A \wedge A^{\circ}$ se completa el sistema $\mathrm{C}_{1}$. Esta negación permite demostrar en $\mathrm{C}_{1}$ la fórmula $((A \rightarrow B) \rightarrow((A \rightarrow$ $\left.\left.\neg^{*} B\right) \rightarrow \neg^{*} A\right)$ ). Sin embargo, en $C_{1}$ el teorema 20 afirma que el esquema $A \leftrightarrow(A \rightarrow$ $B$ ), donde $\mathrm{B}$ es una fórmula cualquiera, trivializa a la teoría, por lo cual la regla $A \leftrightarrow B \Rightarrow \neg A \longleftrightarrow \neg B$ no puede ser válida en $C_{1}$. Por ello, la negación en $C_{1}$ no colapsa en la clásica, sino que evidencia solamente fallas en la simetría. De ahí que, la negación paraconsistente de $\mathrm{C}_{1}$ impide que se satisfaga el principio de sustitución de modo que no siempre será válido el Principio de Reemplazo de Equivalentes, puesto que la negación de fórmulas equivalentes no siempre es equivalente, al igual que en $\mathrm{C}_{\omega}$. Esto equivale en lógica de secuentes a modificar $(\neg \Rightarrow)$.

La mayor diferencia entre $\mathrm{C}_{\omega} \mathrm{y} \mathrm{C}_{1}$ reside en que $\mathrm{C}_{\omega}$ no es finitamente trivializable (da Costa et al. 2007 p.809) mientras que $\mathrm{C}_{1}$ es finitamente trivializable. De hecho, la fórmula $A \wedge \neg^{*} A$ trivializa $C_{1}$ ya que siendo $\neg^{*}$ clásica, entonces se cumple $\Rightarrow$ $\left(A \wedge \neg^{*} A\right) \rightarrow B$ (da Costa et al. 2007: 806). Respecto de la negación fuerte se validan 
todos los teoremas clásicos, es decir siempre que las fórmulas que pudieran generar explosión estén estabilizadas. ${ }^{10}$

\section{La negación en el sistema LP de G. Priest}

Pasemos ahora a analizar la posición realista de G. Priest, llamada por él mismo dialeteismo y expresada esencialmente en su sistema LP y cuyo supuesto filosófico principal consiste en sostener que el mundo real es inconsistente ya que en él existen dialetheias, i.e., proposiciones que son verdaderas y falsas al mismo tiempo las cuales constituyen contradicciones genuinas.

El sistema LP de G. Priest (2001) es un sistema trivalente, ya que contiene un tercer valor de verdad "i". Respecto de las interpretaciones que ha recibido este tercer valor en los sistemas trivalentes, el mismo Priest las agrupa en dos clases. Por un lado, están aquellos sistemas que interpretan este valor como "ni verdadero ni falso", como por ejemplo los sistemas que intentan dar cuenta de los futuros contingentes o de los enunciados que contienen propiedades vagas o conceptos que no denotan. Por el otro lado, están los sistemas que sostienen que hay proposiciones que pueden ser verdaderas y falsas al mismo tiempo. Priest da como ejemplos de estas últimas a las proposiciones "contradictorias" de la tradición hegeliano- marxista, las paradojas de la autorreferencia y ciertas afirmaciones sobre micro objetos de la mecánica cuántica. Precisamente para las conectivas de estas proposiciones Priest propone matrices con un tercer valor, el cual ya no puede ser representado por un lugar vacío, sino por un "atasco" ("glut"), y que se encuentra mejor representado por el valor "01", que significa que las proposiciones atómicas pueden tener el valor verdadero y falso simultáneamente. Los restantes valores tienen el sentido habitual, es decir que el valor 1 debe leerse como verdadero y el valor 0 como falso. Lo más significativo de su sistema está dado por la matriz para la negación ya que ella apareja consecuencias radicales. Su sistema de lógica de las paradojas LP, es el mismo que el sistema K3 de Kleene pero tiene la particularidad de que el conjunto de valores distinguidos es $\{1,01\}$. De esta forma el significado de la negación de LP queda determinado por la siguiente matriz:

\begin{tabular}{c|c}
$A$ & $\neg A$ \\
\hline 0 & 1 \\
01 & 10 \\
1 & 0
\end{tabular}

La matriz característica de LP para el conjunto de las conectivas proposicionales positivas, ${ }^{11}$ conjuntamente con el considerar el valor 01 como valor distinguido hace que la regla ECQ no resulte válida y esto se comprueba cuando en la valuación de la 
premisa $A \wedge \neg A$ se toma el valor 1 de la valuación 01 y el valor 0 de la conclusión $B$. $\mathrm{Si}$ bien este resultado es esperado, el sistema produce otros resultados, algunos de ellos bastante extraños.

(i) El Modus Ponens y la Transitividad (TR) no son inferencias válidas. En efecto MP resulta inválido cuando $V(A)=01$ y $V(B)=0$ y TR $=0$ cuando $V(A)=1$, $V(C)=0$ y $V(B)=01$.

(ii) La contraposición débil $(A \rightarrow B) \rightarrow(\neg B \rightarrow \neg A)$ resultan válida porque su tabla ostenta solamente apariciones de los valores 1 y 01 , y en LP, 01 es un valor distinguido.

(iii) El principio Tercero Excluido y el Principio de No Contradicción resultan válidos, porque su matriz final contiene solo un caso 01 y este es un valor distinguido

(iv) La doble negación $A \leftrightarrow \neg \neg A$. es válida porque su matriz final contiene un caso 01 y éste es valor distinguido

(v) Lo "paradójico" radica en que su sistema permite contradicciones verdaderas. Es decir $A \wedge \neg A$, es verdadera ya que si $V(A)=01$ y $V(\neg A)=01$, entonces, tomando en cuenta el 1 del valor $01, A \wedge \neg A$ resulta con valor $1 \mathrm{y}$, por lo tanto ihay contradicciones verdaderas!

Así, la base deductiva de LP queda establecida por las siguientes reglas: ${ }^{12}$

1) $B \Rightarrow(A \rightarrow B)$

2) $(A \rightarrow B) \wedge(C \rightarrow D) \Rightarrow(A \rightarrow D) \vee(C \rightarrow B)$

3) $A \rightarrow C \Rightarrow(A \wedge B) \rightarrow C$

4) $(A \wedge B) \rightarrow C \Rightarrow(A \rightarrow C) \vee(B \rightarrow C)$

5) $\neg(A \rightarrow B) \Rightarrow A$

6) $\neg A \Rightarrow(A \rightarrow B)$

7) $A \rightarrow B \Rightarrow \neg B \rightarrow \neg A$

8) $\neg \neg A \Rightarrow A$

9) $A \Rightarrow \neg \neg A$

10) $(A \rightarrow B) \wedge(A \rightarrow \neg B) \Rightarrow \neg A(R A b)$

Sin embargo se produce el hecho curioso de que son verdades lógicas (teoremas) pero no inferencias válidas MP, ECQ y el SD.

$$
\begin{aligned}
& \Rightarrow((A \rightarrow B) \wedge A) \rightarrow B) \\
& \Rightarrow(A \wedge \neg A) \rightarrow B \\
& \Rightarrow((A \vee B) \wedge A) \rightarrow B
\end{aligned}
$$

Al igual que otras lógicas subclásicas, en LP el conjunto de las reglas de inferencia o inferencias válidas es menor que el conjunto de verdades lógicas (o teoremas). 
Ahora bien, varias de las reglas de LP requieren de $(\Rightarrow \mathrm{At}),(\neg \Rightarrow)$ y $(\Rightarrow \neg)$, similarmente a lo que sucedía en varios $\mathrm{C}$-sistemas, en particular con $\mathrm{C}_{1}$. Además, la validez de la transposición subminimal necesariamente conduce a aceptar en deducción natural la regla $R A b$ y en lógica de secuentes la regla $(\neg \Rightarrow)$ rechazada en los C-sistemas. Sin embargo, la diferencia fundamental de LP con estos sistemas radica en la falla en LP del MP como regla. Si ahora nos preguntamos qué implica en una deducción no contar con MP como regla de inferencia, estamos en condiciones de responder que se pierde la regla de Eliminación del Condicional en Deducción Natural y consecuentemente con la falla de regla de Introducción del Condicional en el prosecuente $(\rightarrow \Rightarrow)$ en el cálculo de secuentes, pero preservándose ambas reglas de atenuación. Así, es posible afirmar que la caracterización del condicional en tanto regla queda "manca", lo cual a su vez conlleva a afirmar que el condicional de LP no es el condicional material clásico, sino otro tipo de condicional.

En un trabajo anterior C. Oller y G. Palau (2008) propusieron un sistema de secuentes LP (SLP) sin reglas estructurales En él la negación de SLP queda caracterizada por dos de los siguientes tres axiomas:

$$
\begin{aligned}
& \text { Ax.1 } \Gamma, A \Rightarrow \Delta, A, \\
& \text { Ax.2 } \Gamma, \neg A \Rightarrow \Delta, \neg A, \\
& \text { Ax.3 } \Rightarrow \Delta, A, \neg A .
\end{aligned}
$$

Los dos primeros axiomas garantizan la propiedad de Identidad mientras que el tercer axioma compensa la ausencia de las reglas estándares de la negación permitiendo la prueba de TND mediante la regla operatoria de $\Rightarrow \vee$. Dado que LP carece de MP y que $A \rightarrow B$ es entendida como $\neg A \vee B$, la regla operatoria $(\Rightarrow \rightarrow)$ de SLP es sustituida por:

$$
\frac{\Gamma \Rightarrow \Delta, \neg A, B}{\Gamma \Rightarrow A \rightarrow B, \Delta}
$$

Las reglas en SLP que contienen solamente la negación son:

$$
(\neg \neg \Rightarrow) \frac{A, \Gamma \Rightarrow \Delta}{\neg \neg A, \Gamma \Rightarrow \Delta} \quad(\Rightarrow \neg \neg) \quad \frac{\Gamma \Rightarrow \Delta, A}{\Gamma \Rightarrow \Delta, \neg \neg A}
$$

Similarmente a las reglas que A. Avron (2005) enumera como reglas comunes a la negación de varias lógicas subclásicas, en este sistema se emplean reglas para introducir la negación en combinación con otras conectivas, a los efectos de bloquear la derivación en SLP de la regla ECQ y de MP ya que ninguna de ellas permite el pasaje de una fórmula del prosecuente al postsecuente ni del postsecuente al prosecuente. Dado que LP carece de MP, en particular nos interesa destacar las referidas al condicional, ya que ellas se presentan usando la negación: ${ }^{13}$ 


$$
(\neg \rightarrow \Rightarrow) \quad \frac{\Gamma, A, \neg B \Rightarrow \Delta}{\Gamma, \neg(A \supset B) \Rightarrow \Delta} \quad(\Rightarrow \neg \rightarrow) \quad \frac{\Gamma \Rightarrow \Delta, A \quad \Gamma \Rightarrow \Delta, \neg B}{\Gamma \Rightarrow \Delta, \neg(A \supset B)}
$$

Al igual que las reglas presentadas por Avron (2005) la formulación de estas reglas rompe drásticamente la tesis filosófica que hemos propuesto al comienzo de este trabajo, a saber, que el significado de cada conectiva lógica está dado por las reglas de eliminación e introducción correspondientes, las cuales a su vez definen la noción de consecuencia lógica del sistema lógico en cuestión. En síntesis, toda lógica queda determinada por la base deductiva formada por el conjunto de reglas que se satisfacen. En la literatura actual esta tesis ha dado lugar a la propiedad conocida como separabilidad de las conectivas lógicas tanto respecto de sus condiciones de verdad como de las reglas que determinan su uso. Se dice que una base deductiva Q es separable si y sólo si ella permite caracterizar cada conectiva del lenguaje en forma independiente de las restantes. Más precisamente una base deductiva $Q$ es separable si (i) ninguna regla de $\mathrm{Q}$ involucra más de una conectiva y (ii) para cualquier conjunto de conectivas $\mathrm{C}_{i}$ de $\mathrm{Q}$, las consecuencias lógicas de $\mathrm{C}_{i}$ son iguales al conjunto de las consecuencias lógicas de $\mathrm{Q}$ restringidas a las conectivas de $\mathrm{C}_{i}$ (Wójcicki 1998: 183). La base deductiva de la lógica clásica es en efecto separable, tal como queda mostrado tanto en la presentación axiomática de Hilbert, como en los sistemas de deducción natural y de secuentes de Gentzen. Por el contrario, la base deductiva propuesta para LP adolece de ciertas dificultades: 1) No tiene reglas para la negación sino que en su lugar figuran las de Introducción y Eliminación de la Doble Negación y 2) las reglas del condicional (y de las restantes conectivas) involucran para su eliminación o introducción de la negación. Ergo, la base deductiva de LP, no es separable y por lo tanto el significado de sus conectivas no queda unívocamente determinado. Sin embargo, dado que este sistema y en general los sistemas paraconsistentes han sido construidos para dar cuenta de la lógica de dominios vagos o difusos o de los que admiten cierto tipo de contradicciones, que las conectivas lógicas de tales sistemas no tengan la propiedad de separabilidad, no resulta un resultado escandaloso.

\section{Comentarios finales}

Ahora bien, hasta aquí, hemos analizado las propiedades que necesariamente no debe cumplir un operador de negación en un sistema lógico que pretenda tener en cuenta el carácter dialéctico de la realidad. Sin embargo, las leyes sobre la negación rechazadas tanto en los cálculos $\mathrm{C}_{1}, \ldots, \mathrm{C}_{n}, \mathrm{C}_{\omega}$ como en el sistema LP de Priest han generado una negación más débil que la negación clásica, lo cual ha derivado en que muchos lógicos se pregunten si constituyen realmente una negación y nos ha conducido a preguntarnos qué propiedades debe cumplir un operador de negación 
para ser considerado una genuina negación. Cuestión esta que pasaremos ahora a examinar.

En el libro tradicional sobre lógicas multivaluadas de N. Rescher, éste propone una condición semántica para que un operador sea realmente una negación. Tal requisito consiste en prohibir que $A$ y $\neg A$ tengan el mismo valor distinguido. Obviamente, este requisito no es satisfecho por la negación del sistema LP de Priest ya que, como se mostró anteriormente, $A$ y $\neg A$ ambas pueden tener el valor 01 . Por su parte, con la negación del sistema paraconsistente $C_{1}$ de da Costa et al., aunque ésta no sean veritativo funcional, si la $V(A)=0$ entonces $V(\neg A)=1$ (da Costa et al. 2007: 821) y por ello este requisito se cumple parcialmente. En $\mathrm{C}_{\omega}$ sólo se satisface que si $V(\neg \neg A)=1$ entonces $V(A)=1$ lo cual determina que el requisito se cumpla pero respecto de la doble negación. En otras palabras por una u otra razón en los sistemas estudiados $A$ y $\neg A$ no aparecen distinguibles respecto de la verdad en un sentido clásico.

Creemos oportuno preguntarnos entonces a manera de conclusión al igual que Wolfang Lenzen en el trabajo titulado Necessary conditions for negation operators (with particular applications to paraconsistent negation) cuáles son las propiedades esenciales o indispensables para cualquier operador de negación Según este autor, debe cumplir con el siguiente principio:

CP: Si $A$ es al menos tan verdadero como $B$, i.e. si $A$ implica lógicamente a $B$, entonces conversamente $B$ es a lo sumo tan falso como $A$, i.e. la falsedad de $B$ implica lógicamente la falsedad de $A$. En símbolos: Si $A \rightarrow B$ entonces $\neg B \rightarrow$ $\neg A$.

Pedir como condición indispensable (o suficiente) la contraposición parece bastante sensato ya que éste tiene su origen en el esquema de argumento refutatorio empleado por Platón en sus diálogos. Tiene varias formulaciones y la mencionada corresponde a la versión característica de la negación minimal. Además, esta versión es considerada la más débil, ya que su demostración sólo requiere el uso de la regla de Reducción al Absurdo que no implica la doble negación clásica. Ya hemos dicho que la contraposición minimal resulta inválida en el sistema $C_{\omega}$, y sí es válida en $C_{1}$, pero recordando que este resultado se basa en el supuesto de de que la fórmula $B$ esté estabilizada. El sistema dado LP de Priest cumple con este principio, dado que, $(p \rightarrow q) \rightarrow(\neg q \rightarrow \neg p)$ nunca toma el valor 0 y toma el valor 01 en los casos en los que $V(p)=1$ y $V(q)=01, V(p)=01$ y $V(q)=0$ y cuando $V(p)=V(q)=01$. Pero, como el valor 01 es distinguido, entonces el requisito de contraposición es válido y, aún cuando no cumpla con la natural exigencia de Rescher, la negación del sistema LP de Priest aparece como constituyendo una negación genuina, pese a que no posibilite la derivación de ECQ pero sí permita la validez del principio de No Contradicción. Finalmente, dado que tanto $\mathrm{C}_{1}$ como LP cuentan con la validez de la forma 
de Reducción ad absurdum mencionada, es posible concluir que la negación de los sistemas $\mathrm{C}_{1} \mathrm{y}$ LP, aunque no sea la clásica y su formulación en lógica de secuentes tenga los inconvenientes y restricciones señaladas, constituye una genuina negación.

\section{Bibliografía}

Avron, A. 1999. Negation: Two Points of View. In D .Gabbay \& H. Wansing (eds.) What is Negation? Dordrecht: Kluwer Academic Publishers, p. 3-21.

- 2003. Classical Gentzen-type Methods in Propositional Many-Valued Logics. En M. Fitting \& E. Orlowska (eds.) Beyond Two: Theory and Application of Multiple-valued Logic. Heidelberg, New York: Physica-Verlag, p. 117-55.

- 2005. A Non deterministic View on Non-classical Negation. Studia Logica 80: 15994.

Benassi, C. \& Gentilini, P. 2007. Paraconsistent Provability Logic and Rational Epistemic Agents. En J.-Y. Béziau, W. Carnielli, and D. Gabbay (eds.) Handbook of Paraconsistency. Studies in Logic, vol. 9. London: King's College Publications, p. 215-57.

Béziau, J.-Y. 2000. What is paraconsistent logic? Frontiers of Paraconsistent Logic Research Studies Press, p. 95-112.

- 2005. The future of paraconsistent logic. Publicación electrónica: www/logic/Russian/LogStud/02/ls.

- 2007. Adventures in the Paraconsistent Jungle. En J.-Y. Béziau, W. Carnielli, and D. Gabbay (eds.) Handbook of Paraconsistency. Studies in Logic, vol. 9. London: King's College Publications, p. 63-80.

Carnielli, W. A. \& Marcos, J. 2001. A Taxonomy of C-systems. http://arxiv.org/abs/math/0108036.

Carnielli, W. A.; Coniglio, M.; Marcos, J. 2007. Logics of Formal Inconsistency. In D. Gabbay and F. Guenthner (eds.) Handbook of Philosophical Logic, 2nd. ed., vol. 4, p.1-93.

da Costa, N. C. A. \& Wolf, R. 1980. Studies in Paraconsistent Logic I: Dialectical Principle of the Unity of Opposites. Philosophia (Philosophical Quarterly of Israel) IX(2): 189-217.

da Costa, N. C. A.; Krause, D.; Bueno, O. 2007. Paraconsistent Logics and Paraconsistency. In D. M. Gabbay, P. Thagard, and Woods J. (eds.) Handbook of the Philosophy of Science. Philosophy of Logic Volume, Dale Jaquette (ed.) Amsterdam: Elsevier, p. 791-911.

Duran, C. \& Palau, G. 2008. Ley de Peirce, negación y lógica paraconsistente. In C. Duran and A. Hebrard (eds.) Actas de las VI Jornadas de Investigación en Filosofía, vol. II. La Plata, Argentina: Ediciones al Margen, p.207-14.

Gabbay, D. M.; Thagard P; Woods, J. (eds.) 2007. Handbook of the Philosophy of Science. Philosophy of Logic. Amsterdam: North-Holland, Elsevier.

Gabbay, D. \& Gunthner, F. (eds.) 2007. Handbook of the Philosophical Logic, vol. 14. Netherlands: Kluwer Academic Publishers.

Hacking, I. 1994. What is Logic? In D. Gabbay (ed.) What is a logical System? Oxford: Clarendon Press, p. 1-33. Publicado por primera vez en The Journal of Philosophy 6: 285-318.

Hunter, A. 1996. Paraconsistent Logics. In J.-Y. Beziau and W. A. Carnielli (eds.) Paraconsistency with No Frontiers. Studies in Logic and Practical Reasoning vol.4. Amsterdam: North Holland-Elsevier, p. 301-11.

Principia 13(3): 357-70 (2009). 
Lenzen, W. 1998. Necesary conditions for negation operators (with particular applications to paraconsistent negation). In D. Gabbay et al. (eds.) Handbook of Defeasible Reasoning and Uncertainty Management, vol. 2, Reasoning with Potential Contradictions. Dordrecht: Kluwer Academic Publishers, p. 211-35.

Maranhão, J. S. 2001. Some propositional paraconsistent logics. Electronic publication: http://www.uni-leipzig.de/ ${ }^{\circ}$ logik/logikalt/max/nkl2/ks16-10.pdf

Marcos, J. 2003. On negation: Pure local rules. Electronic publication: http://www.cs.math.ist.utl.pt/ftp/pub/MarcosJ/04-M-onplr.pdf

Oller, C. \& Palau, G. 2008. A Sequent System for LP. CLE-e-Prints 8(6).

Priest, G. 1999. What not? A defence of Dialetheic Theory of Negation. In D. Gabbay and H. Wansing (eds.) What is Negation? Dordrecht: Kluwer Academic Publishers, p. 101-20.

Priest, G. 2001. An Introduction to Non- Classical Logic. New York: Cambridge University Press.

Raggio, A. 1968. Propositional sequence-calculi for inconsistent sytems. In A. Raggio (2002), Andrés Raggio. Escritos Completos (1927-1991), Moreno, A. \& Doffi, M. (eds.) Buenos Aires: EUDEBA, p. 179-91.

Rescher, N. 1969. Many-valued logics. New York: McGraw-Hill Company.

Wójcicki, R. 1988. Theory of Logical Calculi, Basic Theory of Consequence Operations. Dordrecht: Kluwer Academic Publishers.

Gladys Palau

Departamento de Filosofía

Facultad de Humanidades y Ciencias de la Educación (FaHCE)

Universidad Nacional de La Plata (UNLP)

Universidad de Buenos Aires

Argentina

gadi1@fibertel.com.ar

Cecilia Duran

Departamento de Filosofía

Facultad de Humanidades y Ciencias de la Educación (FaHCE)

Universidad Nacional de La Plata (UNLP)

Argentina

cdurancduran@gmail.com

Resumo. Neste trabalho concorda-se com a tese de I. Hacking segundo a qual o significado das constantes lógicas é dado pelas Regras de Introdução e Eliminação do cálculo de sequentes de Gentzen que caracterizam a concepção da noção de consequência lógica abstrata. Perguntamos quais são as regras mínimas que um conectivo deve satisfazer para que seja considerado uma negação genuína. Tomaremos como referência para tratar dessa questão os C-sistemas de Newton da Costa e o sistema LP de Graham Priest. Finalmente, analisaremos esses sistemas na lógica de sequentes a fim de mostrar que a negação paraconsistente ou bem carece das regras puras de eliminação e negação da negação ou ela envolve outros conectivos, o que torna difícil atribuir um significado unívoco à negação paraconsistente.

Palavras-chave: Lógica paraconsistente, negação, cálculo de sequentes.

Principia 13(3): 357-70 (2009). 


\section{Notas}

${ }^{1}$ La demostración primera se debe en realidad a Gödel, quien demuestra que todas las leyes clásicas expresadas en términos de la conjunción y negación son leyes intuicionistas y la segunda a Anderson y Belnap (1975) según quienes las leyes clásicas expresadas con conjunción, disyunción y negación son todas leyes relevantistas.

${ }^{2}$ En la literatura sobre el tema también se menciona que la lógica paraconsistente no cumple $\Gamma, A, \neg A \Rightarrow \Delta$, denominada Explosión (o Pseudo Scotus), pero en el texto nos referiremos siempre a ECQ ya que ellas no se distinguen en una lógica de conclusión única.

${ }^{3}$ Hay otra, de origen medieval, que en deducción Natural utiliza Adición y Silogismo Disyuntivo y que su demostración en secuentes involucra también la regla $(\neg \Rightarrow)$.

${ }^{4}$ En Carnielli y Marcos (2001) la Ley de Dummett, se agrega al conjunto de axiomas de $\mathrm{C}_{\omega}$ para obtener Cmin (considerado por ellos el más débil de la jerarquía de los C-sistemas).

${ }^{5}$ Otra razón para rechazar Reductio ad absurdum reside en que esta regla permite derivar reglas de la negación clásica que harían colapsar la negación paraconsistente con la clásica.

${ }^{6} \mathrm{El}$ rechazo de SD conduce a un resultado paradójico ya que en los C-sistemas no es posible derivar la fórmula $B$ por $\mathrm{SD}$, pero sí puede derivarse a partir de $((A \rightarrow B) \wedge A)$ por MP, lo cual implica el rechazo de la definición del condicional en términos de disyunción y negación.

${ }^{7}$ Como se señala en Benassi y Gentilini (2007: 222), si esto no fuera posible, tampoco podría demostrarse $\neg A \Rightarrow \neg A$ a partir de $A \Rightarrow A$. También se requiere de este recurso para derivar $(A \rightarrow \neg A) \rightarrow \neg A$.

${ }^{8}$ Dicha negación permite definir una trivialización parcial (o positiva).

9 También se puede optar por agregarla a las ya dadas como regla derivada:

$$
\frac{B^{\circ}, \Gamma_{1}, A \rightarrow B \Rightarrow \Delta_{1} \quad B^{\circ}, \Gamma_{2}, A \rightarrow \neg B \Rightarrow \Delta_{2}}{\Gamma_{1}, \Gamma_{2} \Rightarrow \neg A, \Delta_{1}, \Delta_{2}}
$$

${ }^{10}$ Otros análisis (Carnielli et al. 2007) se han dedicado al detallado análisis de los sistemas de la Lógica de la Inconsistencia Formal determinando que ya no es posible identificar a los C-sistemas con los sistemas $C_{n}$ de da Costa sino que abarcan un vastísimo campo de sistemas paraconsistentes dentro de los cuales es posible expresar consistencia.

${ }^{11}$ La matriz de Priest para las restantes conectivas es la siguiente:

\begin{tabular}{|c|c|c|c|c|c|c|c|c|c|c|}
\hline \multirow[b]{2}{*}{ LP } & \multicolumn{4}{|c|}{$A \wedge B$} & \multicolumn{3}{|c|}{$A \vee B$} & \multicolumn{3}{|c|}{$A \rightarrow B$} \\
\hline & $A$ & 1 & 01 & 0 & 1 & 01 & 0 & 1 & 01 & 0 \\
\hline & 1 & 1 & 01 & 0 & 1 & 1 & 1 & 1 & 01 & 0 \\
\hline & 01 & 01 & 01 & 0 & 1 & 01 & 01 & 1 & 01 & 01 \\
\hline & 0 & 0 & 0 & 0 & 1 & 01 & 0 & 1 & 1 & 1 \\
\hline
\end{tabular}

${ }^{12} \mathrm{Si}$ se quiere la validez MP como regla de inferencia, hay que cambiar la matriz del condicional y se obtendría el sistema RM3.

${ }^{13}$ Las reglas propuestas para las restantes conectivas son:

$$
\begin{aligned}
& (\neg \wedge \Rightarrow) \quad \frac{\Gamma, \neg A \Rightarrow \Delta \quad \Gamma, \neg B \Rightarrow \Delta}{\Gamma, \neg(A \wedge B) \Rightarrow \Delta} \quad(\Rightarrow \neg \wedge) \quad \frac{\Gamma \Rightarrow \Delta, \neg A, \neg B}{\Rightarrow \Delta, \neg(A \wedge B)} \\
& (\neg \vee \Rightarrow) \frac{\Gamma, \neg A, \neg B \Rightarrow \Delta}{\Gamma, \neg(A \vee B) \Rightarrow \Delta} \quad(\Rightarrow \neg \vee) \frac{\Gamma \Rightarrow \Delta, A \Gamma \Rightarrow \Delta, B}{\Gamma \Rightarrow \Delta, \neg(A \vee B)}
\end{aligned}
$$

Principia 13(3): 357-70 (2009). 